\title{
Lab Lupus Inhibitor Test
}

National Cancer Institute

\section{Source}

National Cancer Institute. Lab Lupus Inhibitor Test. NCI Thesaurus. Code C70648.

Laboratory test to determine whether a lupus anticoagulant is present in the subject.

Presence of a lupus inhibitor is most often detected by prolong ation of the activated partial thromboplastin time. Presence of a lupus anticoagulant may actually increase risk for blood clots, and may indicate presence of systemic lupus. See definition of lupus anticoagulant for more details. 